\title{
Duodenal obstruction due to annular pancreas associated with carcinoma of the duodenum
}

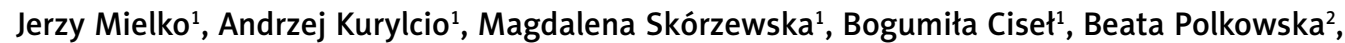 \\ Karol Rawicz-Pruszyński ${ }^{1}$, Jadwiga Sierocińska-Sawa ${ }^{3}$, Wojciech P. Polkowski ${ }^{1}$ \\ ${ }^{1}$ Department of Surgical Oncology, Medical University of Lublin, Lublin, Poland \\ ${ }^{2}$ Student Research Group, Medical University of Lublin, Lublin, Poland \\ ${ }^{3}$ Department of Pathology, University Hospital No. 1, Lublin, Poland
}

Gastroenterology Rev 2016; 11 (2): 139-142

DOI: $10.5114 / p g .2016 .57885$

Address for correspondence: Jerzy Mielko MD, PhD, Department of Surgical Oncology, Medical University of Lublin, $11 \mathrm{~S}$. Staszica St, 20-081 Lublin, Poland, phone: +48 8153443 13, fax: +48 8153223 95, e-mail: jerzy.mielko@umlub.pl

Annular pancreas, the second most common anomaly of the pancreas, is the development of a ring of pancreatic tissue that surrounds and often embraces the duodenum. It was first described by Tiedelmann in 1818 and named "annular pancreas" by Ecker in 1862 [1]. It is usually present in childhood, with symptoms due to duodenal obstruction. In 50\% of cases, clinical manifestations become visible after childhood, with abdominal pain, chronic duodenal obstruction, peptic ulceration, obstructive jaundice, pancreatitis, and pancreatic head mass [2, 3]. Therefore, duodeno-jejunostomy is usually applied to relieve strictures caused by such an annulus. Bypass surgery is the treatment of choice [4].

Primary duodenal carcinoma is rare and represents $0.3 \%$ of all gastrointestinal malignancies and $25-35 \%$ of malignant tumours of the small intestine $[5,6]$. Resectability and the presence of distant metastatic disease are the strongest determinants of outcome for patients with duodenal adenocarcinoma. In cases of cancers of

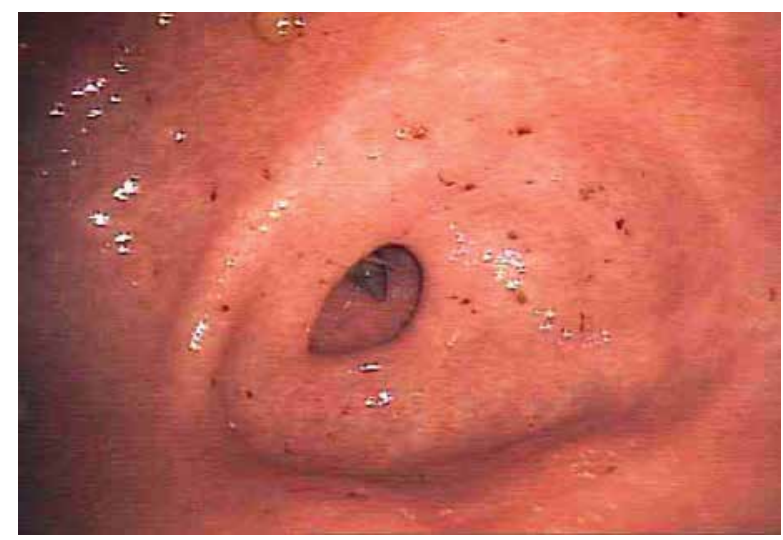

Figure 1. Endoscopic view of the duodenum showing ulceration with duodenal stenosis at the second portion the first and second part of the duodenum, the most common procedure is pancreaticoduodenectomy. Despite advancements in techniques of diagnosis and resection and decreased perioperative mortality and morbidity, 5-year survival varies from $37 \%$ [7] to 57\% [8].

Malignancy in the setting of annular pancreas is an unusual event, and hence only a few cases associated with pancreatic carcinoma, ampullary carcinoma, and cholangiocarcinoma have been reported [9-13]. Only 1 case of annular pancreas associated with duodenal carcinoma has been reported, but without description of the follow-up [14]. We report a case of duodenal carcinoma in a patient with an annular pancreas treated with radical surgery with complete follow-up data.

A 53-year-old lady suffered from nausea, postprandial fullness, and vomiting, and weight loss. She was diagnosed (endoscopy Figure 1, CT scan of the abdomen Figure 2) to have duodenal obstruction with gastric bezoar. The upper intestinal contrast study

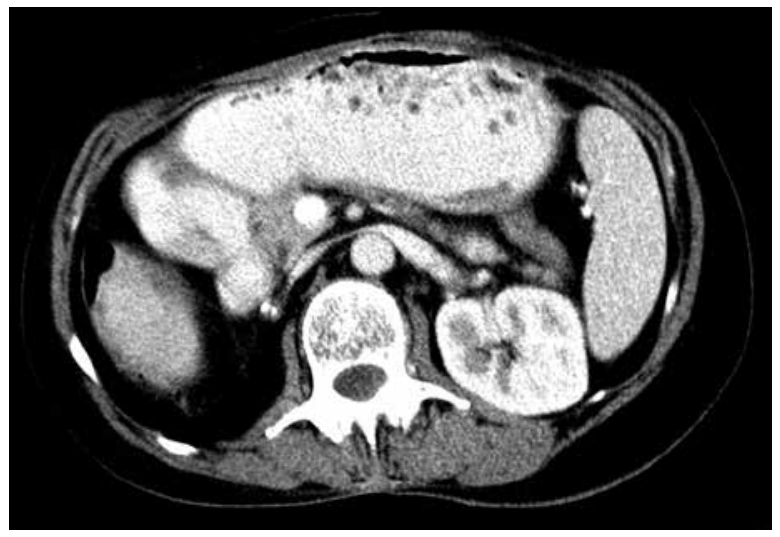

Figure 2. Computed tomography scan image revealing a significant dilatation of the stomach with bezoar and the proximal duodenum 


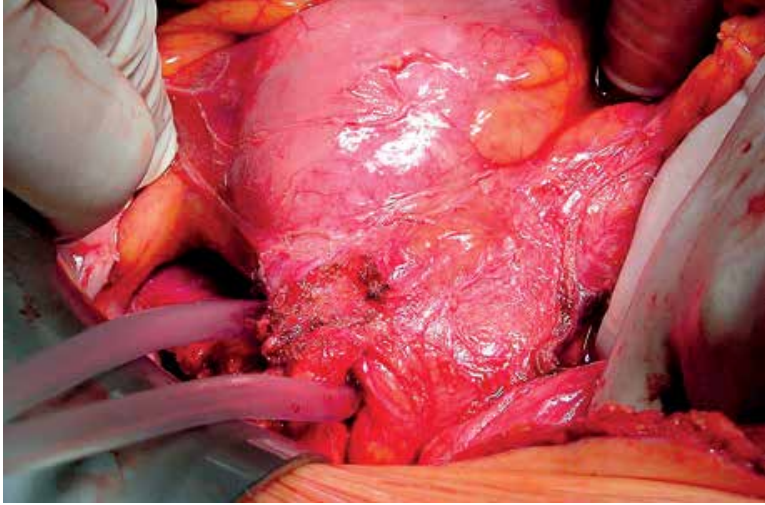

Figure 3. Intraoperative view of the annular pancreas encircling the second part of the duodenum

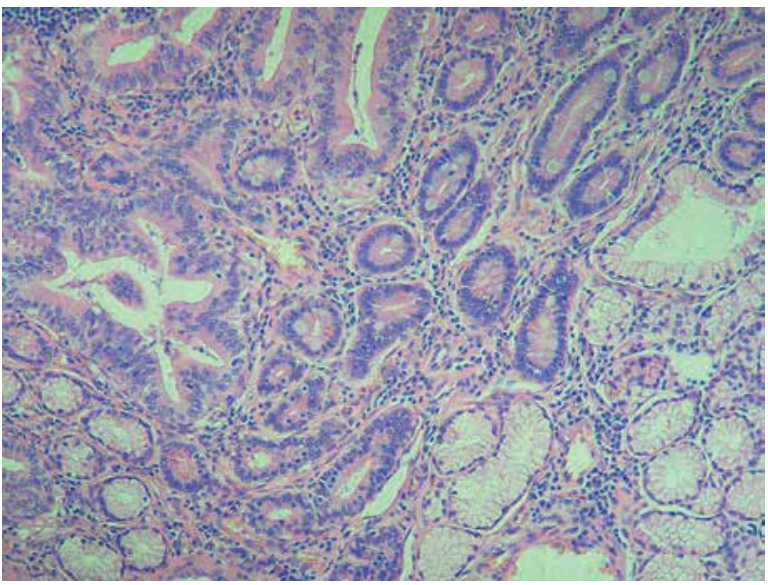

Figure 5. Tubular adenocarcinoma of the duodenum. Neoplastic tubules border on normal duodenal glands (four-micron sections of representative blocs of the lesion were stained with haematoxylin and eosin $(H+E) ; 100 x)$

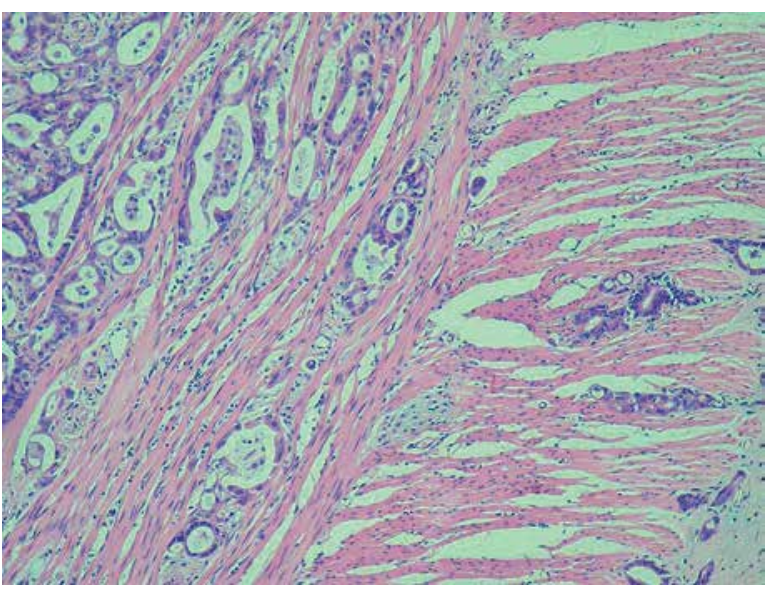

Figure 7. Neoplastic infiltrate of the muscular coat of the duodenum encompasses both an inner circular layer and an outer longitudinal layer $(H+E ; 100 \times)$

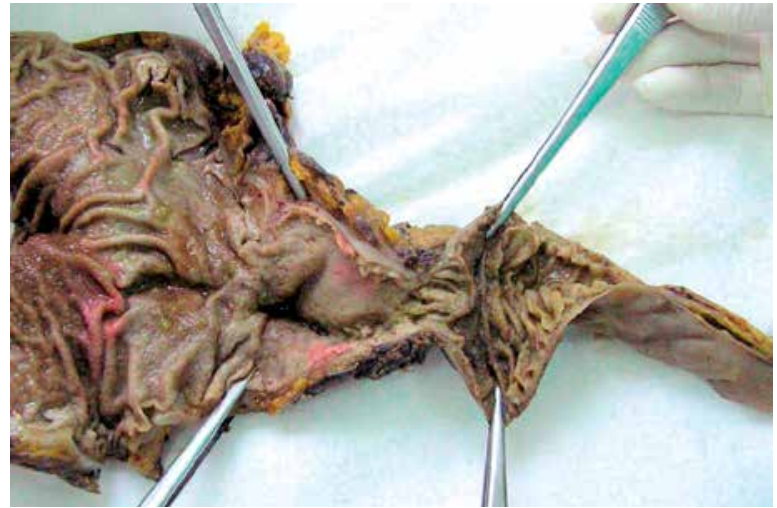

Figure 4. Cross-sectional view showing the duodenum surrounded by the pancreas tissue (annular pancreas)

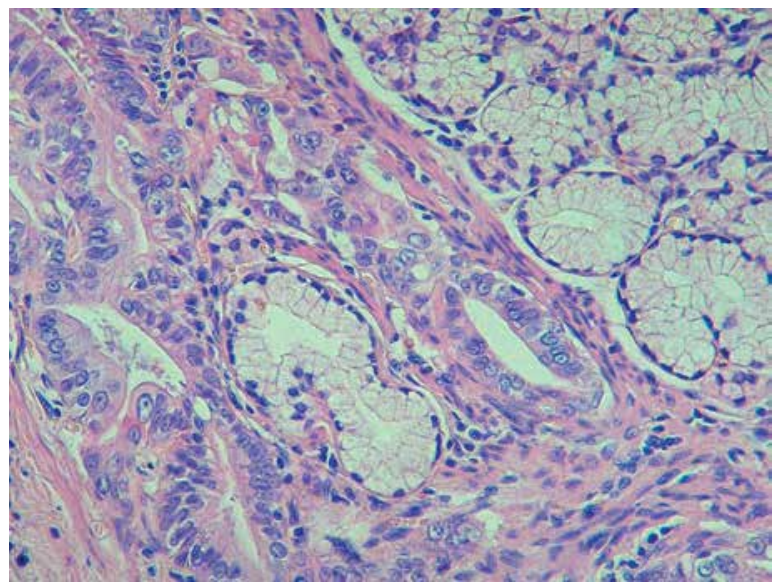

Figure 6. Tubular adenocarcinoma of the duodenum. Neoplastic tubules border on normal duodenal glands (four-micron sections of representative blocs of the lesion were stained with haematoxylin and eosin $(H+E) ; 200 x)$

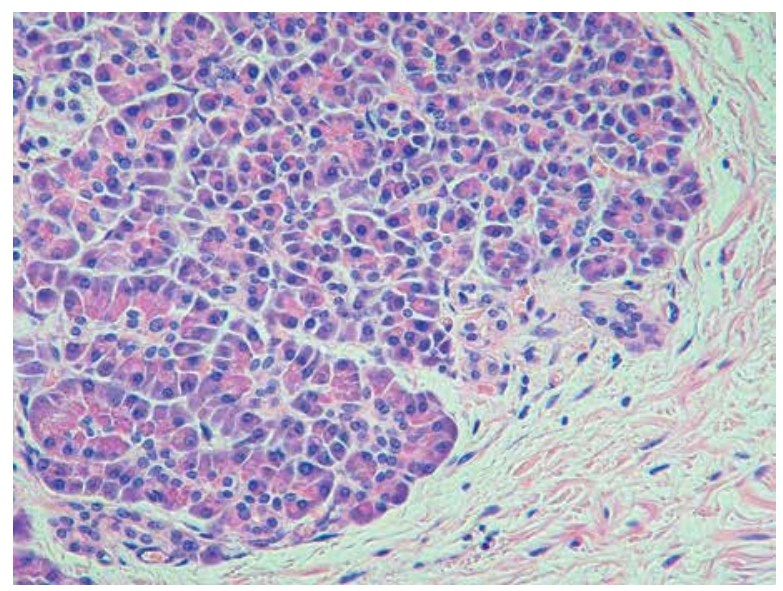

Figure 8. Fragment of the pancreas (annular pancreas), which is not involved in cancerous infiltrate $(H+E ; 100 x)$ 
findings of annular filling defect in the duodenum, prestenotic dilatation, and reverse peristalsis in the proximal segment were suggestive of annular pancreas. A pathology report of the endoscopic biopsy material taken from the stenotic part of the duodenum revealed suspicion of malignancy, i.e. atypic cells. The patient was scheduled for a laparotomy with intraoperative pathological investigation of the suspected lesion, and if malignancy is proven a pancreaticoduodenectomy is anticipated. During the operation a band of fibrous (probably pancreatic) tissue of hard texture encircling the second part of the duodenum was found (Figure 3). Division of the pancreatic ring was done and the abnormal tissue sent for immediate pathology. Once malignant diagnosis was confirmed, and classical Kausch-Whiple pancreaticoduodenectomy with standard lymphadenectomy was done without perioperative complications. The resection specimen had been routinely fixed in buffered $10 \%$ formaldehyde and embedded in paraffin wax (Figure 4). Operative time was $300 \mathrm{~min}$, and blood loss was $420 \mathrm{ml}$. The postoperative course was uneventful; therefore, the patient was discharged from the hospital on the sixteenth postoperative day. A definitive pathological report confirmed tubular carcinoma (G2) of the duodenum associated with the annular pancreas with no lymph node involvement (pT2NOMO) (Figures 5-8). No adjuvant chemotherapy was administered. The patient was followed-up every 3 months thereafter for the first 3 years. In the first (2008) and second year (2009) of the follow-up, abdominal CT-scan was done without signs of recurrence. After 54 months the patient became heavily jaundiced and cachectic, and was therefore hospitalised. Advanced loco-regional recurrence was found on MR cholangiogram, with no liver metastases. The patient died, and no autopsy was performed.

Annular pancreas is a rare congenital abnormality, and in adult patients it presents with clinical features that differ from those seen in newborns [15]. Duodenal obstruction is a rare symptom of annular pancreas in adults. It is necessary to remember about the coexistence of the annular pancreas when patients with pancreatic or periampullary malignancies are complicated with unexpected obstruction of the second portion of the duodenum in proportion to the size [9]. Features in the adult patient include peptic ulceration, duodenal obstruction, acute pancreatitis, and obstructive jaundice. Treatment strategies for annular pancreas with obstructive jaundice remain controversial, but usually pancreaticoduodenectomy is necessary [2]. In adult patients pancreatic head malignancy usually produces jaundice, and more rarely duodenal obstruction. Nearly always the main cause of this clinical picture is pancreatic head carcinoma, but pancreas annulare should be taken into consideration. The duodenal ring should always be verified on pathological examination since it may harbour this rare type of adenocarcinoma.

In adult patients with duodenal obstruction due to annular pancreas, consideration of associated or coexisting duodenal malignancy is mandatory. Only radical surgery (pancreaticoduodenectomy) may provide longterm survival in such cases.

\section{Conflict of interest}

The authors declare no conflict of interest.

\section{References}

1. Kiernan PD, ReMine SG, Kiernan PC, ReMine WH. Annular pancreas: May Clinic experience from 1957 to 1976 with review of the literature. Arch Surg 1980; 115: 46-50.

2. Shan YS, Sy ED, Lin PW. Annular pancreas with obstructive jaundice: beware of underlying neoplasm. Pancreas 2002; 25 : 314-6.

3. Urayama S, Kozarek R, Ball T, et al. Presentation and treatment of annular pancreas in an adult population. Am J Gastroenterol 1995; 90: 995-9.

4. Thomford NR, Knight PR, Pace WG, Madura JA. Annular pancreas in the adult: selection of operation. Ann Surg 1972; 176 : 159-62.

5. Sohn TA, Lillemoe KD, Cameron JL, et al. Adenocarcinoma of the duodenum: factors influencing long-term survival. J Gastrointest Surg 1998; 2: 79-87.

6. Barnes G Jr, Romero L, Hess KR, Curley SA. Primary adenocarcinoma of the duodenum: management and survival in 67 patients. Ann Surg Oncol 1994; 1: 73-8.

7. Onkendi EO, Boostrom SY, Sarr MG, et al. 15-year experience with surgical treatment of duodenal carcinoma: a comparison of periampullary and extra-ampullary duodenal carcinomas. J Gastrointest Surg 2012; 16: 682-91.

8. Ohigashi $\mathrm{H}$, Ishikawa O, Tamura S, et al. Pancreatic invasion as the prognostic indicator of duodenal adenocarcinoma treated by pancreatoduodenectomy plus extended lymphadenectomy. Surgery 1998; 124: 510-5.

9. Yasui A, Nimura Y, Kondou S, Kamiya J. Duodenal obstruction due to annular pancreas associated with pancreatic head carcinoma. Hepatogastroenterology 1995; 42: 1017-22.

10. Rathnaraj S, Singh SK, Verghese M. Annular pancreas associated with carcinoma of papilla. Indian J Gastroenterol 1998; 17: 110 .

11. Kamisawa T, Tabata I, Isawa T, et al. Annular pancreas associated with carcinoma in the dorsal part of pancreas divisum. Int J Pancreatol 1995; 17: 207-11.

12. Foo FJ, Gill U, Verbeke CS, et al. Ampullary carcinoma associated with an annular pancreas. JOP 2007; 8: 50-4.

13. Cholet F, Bideau K, Nonent M, et al. Coexistence of annular pancreas with carcinoma in the dorsal part of pancreas divisum: diagnostic value of magnetic resonance cholangiopancreatography. Abdom Imaging 2004; 29: 703-6. 
14. Bronnimann E, Potthast S, Vlajnic T, et al. Annular pancreas associated with duodenal carcinoma. World J Gastroenterol 2010; 16: 3206-10.

15. Zyromski NJ, Sandoval JA, Pitt HA, et al. Annular pancreas: dramatic differences between children and adults. J Am Coll Surg 2008; 206: 1019-25.

Received: 13.04 .2015

Accepted: 28.06.2015 